\title{
Conduta cirúrgica na subluxação do cristalino
}

\author{
Surgical approach to the subluxated lens
}

\author{
Nórton Souto Severo ${ }^{1}$ \\ Fabíola Kleinert ${ }^{2}$ \\ Sérgio Kwitko ${ }^{3}$
}

\begin{tabular}{|c|}
\hline RESUMO \\
\hline $\begin{array}{l}\text { Objetivo: Apresentar } 10 \text { casos de subluxação de cristalino, secundários a } \\
\text { trauma ou a síndrome de Marfan, discutindo o tratamento e os resultados } \\
\text { obtidos. Métodos: Foram estudados } 10 \text { olhos de } 9 \text { pacientes, } 6 \text { casos } \\
\text { secundários a trauma contuso e } 4 \text { casos a síndrome de Marfan. A idade } \\
\text { média foi de } 48,9 \pm 18,2 \text { anos, com tempo de seguimento de } 34,6 \pm 11,6 \text { meses } \\
\text { (12 a } 62 \text { meses). Os pacientes foram submetidos à facectomiaintracapsular } \\
\text { (1 caso), extracapsular com capsulorrexe ( } 1 \text { caso), facoemulsificação sem } \\
\text { LIO ( } 1 \text { caso), e facoemulsificação com implante de LIO ( } 7 \text { casos). Anéis } \\
\text { expansores capsulares foram utilizados em } 7 \text { olhos. Resultados: Houve } \\
\text { melhora da acuidade visual em todos os casos durante o seguimento, } \\
\text { exceto em um, em que houve descolamento de retina, } 20 \text { dias após o } \\
\text { procedimento. Conclusão: A facectomia comimplante de LIO empacientes } \\
\text { com cristalino subluxado é procedimento viável, principalmente com a } \\
\text { facoemulsificação e o uso do anel expansor capsular, podendo melhorar } \\
\text { bastante a acuidade visual e a qualidade de vida desses pacientes. }\end{array}$ \\
\hline
\end{tabular}

Descritores: Subluxação do cristalino/cirurgia; Traumatismos oculares; Síndrome de Marfan; Extração de catarata

\section{INTRODUÇÃO}

A subluxação do cristalino, familiar ou adquirida, apresenta-se sempre como um desafio terapêutico. De um lado, temos olhos estruturalmente frágeis, que normalmente apresentam outras condições que merecem atenção, como degenerações retinianas em pacientes com síndrome de $\operatorname{Marfan}^{(1)}$ ou alterações traumáticas do segmento anterior em casos pós-trauma ${ }^{(2)}$, apenas para citar dois exemplos; de outro, a necessidade de oferecermos a estes pacientes a melhor opção disponível, buscando a plena reabilitação visual.

O cristalino subluxado pode ser assintomático, provocar astigmatismo leve, distorção óptica pela presença da borda equatorial no eixo visual, glaucoma, uveíte, e até ambliopia em casos congênitos ${ }^{(1)}$. Tem sido associado com várias síndromes, sendo a mais freqüente a síndrome de Marfan, em que até $80 \%$ dos casos apresentam algum grau de subluxação do cristalino, tipicamente superior e temporal ${ }^{(3)}$. Outras, menos prevalentes, como as síndromes de Weill-Marchesani, de Stickler e de Ehlers-Danlos, homocistinúria, hiperlisinemia e aniridia, também podem apresentar subluxação ${ }^{(1,4)}$. Condições adquiridas, como trauma e tumores de úvea anterior também são responsáveis por uma parcela dos casos de ectopia cristaliniana ${ }^{(3)}$. Nesses pacientes, além da subluxação, podem ser encontradas alterações do segmento anterior, como recesso de ângulo, glaucoma, ciclodiálise, ruptura de cápsula posterior, catarata traumática, entre outros ${ }^{(5)}$.

A conduta, diante de casos tecnicamente tão difíceis, tem sido classicamente a observação e a correção com meios ópticos, como óculos ou lentes de contato, quando possível ${ }^{(1)}$. e Doenças Externas do Serviço de Oftalmologia do Hospital de Clínicas de Porto Alegre.

${ }^{2}$ Acadêmica de Medicina da Universidade Federal do Rio Grande do Sul - UFRGS.

Médico do Setor de Córnea do Serviço de Oftalmologia do Hospital de Clínicas de Porto Alegre. Mestre e Doutor em Oftalmologia. Rua São Caetano, 79 - São Leopoldo (RS) CEP 93.010-090

E-mail: eyenet@bol.com.br

Recebido para publicação em 17.02.2003

Versão revisada recebida em 29.07.2003

Aprovação em 07.08.2003 
Medidas mais intervencionistas, como a lensectomia via pars-plana, a facectomia intracapsular, a zonulólise, e mesmo a facectomia extracapsular sem implante de lente intra-ocular (LIO) apresentam resultados apenas razoáveis nestes pacientes ${ }^{(3,5)}$.

O desenvolvimento da facoemulsificação e o constante aprimoramento da técnica cirúrgica permitiram que novas propostas de tratamento surgissem para esses casos. O implante de LIO de câmara posterior com fixação escleral passou a ser uma opção viável de reabilitação visual, com a possibilidade de colocação da LIO em localização quase fisiológica, embora este procedimento ainda estivesse longe de ser considerado o ideal, em olhos em que qualquer trauma ou manipulação adicional é potencialmente perigoso ${ }^{(6-7)}$.

Em 1993, Witschel e Legler propuseram o implante de um anel intracapsular que, fixado ou não ao sulco ciliar, permitiria a estabilização da cápsula posterior e o implante de uma LIO adequada $^{(8)}$. Cionni e Osher, em 1998, acrescentaram ao anel intracapsular uma alça, que passou a permitir a fixação do saco capsular com manutenção de sua integridade, tornando ainda mais fisiológica uma cirurgia que evoluiu muito nos últimos 5 $\operatorname{anos}^{(9)}$.

São apresentados 10 casos de subluxação de cristalino, secundária a trauma ou a síndrome de Marfan, e o tratamento realizado e os resultados obtidos nestes pacientes são discutidos.

\section{MÉTODOS}

Analisamos, retrospectivamente, 10 olhos de 9 pacientes com subluxação de cristalino submetidos a tratamento cirúrgico, no período de abril de 1997 a junho de 2001.

Os pacientes, antes da cirurgia, foram submetidos à avaliação oftalmológica completa, que incluiu medida da acuidade visual corrigida e não corrigida, tonometria, biomicroscopia, mensuração da área de subluxação (em graus, no microscópio cirúrgico) e avaliação da periferia da retina.

Todos os pacientes foram operados pelo mesmo cirurgião (S.K.), sob anestesia local peri-bulbar, com exceção de um paciente com 7 anos de idade, que foi submetido à anestesia geral.

Facectomia intracapsular com implante de LIO através de fixação escleral no sulco ciliar em 1 caso; facectomia extracapsular com implante de LIO no sulco ciliar em 1 caso; facoemulsificação sem implante de LIO em 1 caso; e facoemulsificação com implante de LIO em 7 casos, com 2 implantes no sulco ciliar, e 5 implantes de LIO no saco capsular. $\mathrm{O}$ anel expansor capsular (Mediphacos ou Morscher)foi utilizado em 7 casos.

Não utilizamos anel expansor capsular no caso submetido a facectomia intracapsular, no caso submetido a facectomia extracapsular e em um dos casos cuja LIO foi implantada no sulco ciliar.

No pós-operatório, foram avaliados a reação inflamatória à biomicroscopia, o posicionamento da LIO, a melhora da acuidade visual com correção (c/c), além das complicações trans e pós-operatórias.

\section{RESULTADOS}

Foram tratados, no período entre abril de 1997 e junho de 2001, 10 olhos de 9 pacientes com subluxação de cristalino e perda importante de acuidade visual. Eram 3 homens ( 4 olhos) e 6 mulheres ( 6 olhos), com idade média de 48,9 $\pm 18,2$ anos, e diagnóstico de síndrome de Marfan em 3 pacientes (4 olhos) e de subluxação traumática em 6 casos.

O período de seguimento foi de 34,6 6 11,6 meses ( 12 a 62 meses).

A heterogeneidade de achados pré-operatórios e das condutas cirúrgicas adotadas impede uma análise uniforme dos pacientes. Assim, apresentaremos, de maneira sucinta, os casos estudados (Tabela 1).

\section{DISCUSSÃO}

Síndrome de Marfan e trauma contuso são duas causas importantes de subluxação cristaliniana, respondendo pela grande maioria dos casos congênitos e adquiridos, respectivamente $^{(1,5)}$. A abordagem terapêutica é complexa, pois são olhos em que a integridade estrutural não é a comumente encontrada em pacientes com alterações cristalinianas com indicação de facectomia. Recesso de ângulo, ciclodiálise, ruptura zonular parcial e mesmo ruptura primária de cápsula posterior são achados freqüentes em pacientes com subluxação de origem traumática, piorando o prognóstico visual e tornando qualquer procedimento tecnicamente mais difícil ${ }^{(5)}$. Já pacientes com subluxação congênita, classicamente por síndrome de Marfan, apresentam uma miríade de achados oculares associados, que vão de alterações estruturais de ângulo à maior incidência de degenerações retinianas periféricas, com risco de ruptura e descolamento de retina regmatogênico (como no caso 2$)^{(3-4)}$.

Vários tratamentos, ao longo dos anos, têm sido sugeridos para pacientes nessas condições. Desde a simples observação, para casos assintomáticos ou com alterações ópticas corrigíveis com óculos ou lentes de contato, até procedimentos mais invasivos, como lensectomia via pars-plana sem implante de LIO ou zonulólise enzimática da porção luxada da cápsula com a intenção de deslocar o cristalino do eixo visual, estabelecendo uma condição de pseudo-afacia ${ }^{(1,5)}$.

Na última década, com o impressionante avanço da técnica de facectomia com a facoemulsificação, da biocompatibilidade e desenho das lentes intra-oculares e dos recursos disponíveis como adjuvantes da cirurgia (fixação escleral de LIO e anel expansor capsular), a facectomia em olhos com cristalino subluxado com implante primário de LIO passou a ser uma opção viável, levando inclusive àqueles pacientes com acuidade visual razoável no pré-operatório (caso 1, síndrome de Marfan) a possibilidade de melhora com a cirurgia (no caso citado, houve melhora de 20/60 para 20/25) ${ }^{(7,10-12)}$.

Em 1993, Witschel e Legler sugeriram o uso de um anel expansor endocapsular, com o objetivo de estabilizar o saco 


\begin{tabular}{|c|c|c|c|c|c|c|c|c|}
\hline Caso & Sexo & Idade & Causa & $\begin{array}{l}\text { Subluxação } \\
\text { (ângulo) }\end{array}$ & AV pré & AV pós & Cirurgia & Observações \\
\hline 1 & $\mathrm{H}$ & 65 & trauma & 180 & CD $50 \mathrm{~cm}$ & $20 / 200$ & $\begin{array}{l}\text { FIC+TREC+LIO } \\
\text { c/fixação }\end{array}$ & $\begin{array}{l}\text { glaucoma secundário, } \\
\text { controle clínico }\end{array}$ \\
\hline 2 & $\mathrm{H}$ & 70 & trauma & 120 & $20 / 70$ & $20 / 40$ & FEC+LIO c/fixação & $\begin{array}{l}\text { glaucoma secundário, } \\
\text { controle clínico - } \\
\text { OCP no seguimento }\end{array}$ \\
\hline 3 & M & 54 & trauma & 90 & PL & MM & Faco+anel+LIO & $\begin{array}{l}\text { DR nasal, retinopexia/endolaser } \\
\text { simultâneos, retina aplicada }\end{array}$ \\
\hline 4 & M & 46 & trauma & 90 & $20 / 80$ & $20 / 25$ & $\mathrm{Faco}+$ anel+LIO & \\
\hline 5 & M & 66 & trauma & 100 & CD $3 \mathrm{~m}$ & $20 / 40$ & $\begin{array}{l}\text { Faco+anel+ } \\
\mathrm{LIO}+\text { vitrec anterior }\end{array}$ & \\
\hline 6 & M & 40 & trauma & 120 & CD $2 \mathrm{~m}$ & $20 / 20$ & $\begin{array}{l}\text { Faco+anel+ } \\
\mathrm{LIO+vitrec} \text { anterior }\end{array}$ & \\
\hline 7 & M & 53 & Marfan & 180 & $20 / 60$ & $20 / 25$ & $\mathrm{Faco}+$ anel+LIO & \\
\hline 8 & M & 39 & Marfan & 180 & CD $1 \mathrm{~m}$ & MM & Faco+anel & DR regmatogênico \\
\hline 9 & $\mathrm{H}$ & 7 & Marfan & 270 & $20 / 400$ & $20 / 40$ & Faco+anel+LIO & \\
\hline 10 & $\mathrm{H}$ & 7 & Marfan & 270 & $20 / 400$ & $20 / 40$ & $\mathrm{Faco}+\mathrm{anel}+\mathrm{LIO}$ & \\
\hline \multicolumn{9}{|c|}{$\begin{array}{l}\text { H - homem; M - mulher; AV - acuidade visual corrigida; pré - pré-operatório; pós - pós-operatório; CD - conta dedos; PL - percepção luminosa; MM - movimento de } \\
\text { mão; DR - descolamento de retina; OCP - opacificação da cápsula posterior; FIC - facectomia intracapsular; FEC - facectomia extracapsular; Faco - facoemulsificação; } \\
\text { TREC - trabeculectomia; LIO - lente intra-ocular; vitrec - vitrectomia }\end{array}$} \\
\hline
\end{tabular}

capsular e permitir uma facoemulsificação segura e o implante de LIO em posição adequada ${ }^{(8)}$. Este recurso, bom para casos com perda moderada do suporte zonular, se mostrou insuficiente para pacientes com diálise ou fraqueza severas da zônula, quando então o anel deve ser fixado à esclera, na área correspondente à subluxação ${ }^{(7,9-10,13)}$. Cionni e Osher, em 1998 apresentaram um anel endocapsular que permite a fixação escleral sem violação do saco capsular, com excelente suporte e centralização durante a cirurgia e durante o acompanhamento pósoperatório $^{(9)}$.

Nossa série espelhou bem a diversidade de condutas que podem ser utilizadas em casos de subluxação. Independente do tratamento utilizado - facectomia intra ou extracapsular, facoemulsificação, implante primário, anel expansor - obtivemos melhora da acuidade visual corrigida em 9 dos 10 (90\%) casos. Houve uma complicação pós-operatória, um descolamento regmatogênico de retina, em paciente com síndrome de Marfan. Este achado é consistente com a literatura, que aponta esta como uma das complicações mais freqüentes nestes pacientes, de tratamento difícil, comprometendo o resultado de qualquer procedimento cirúrgico ${ }^{(4)}$.

Nossos resultados sugerem que a facectomia em pacientes com cristalino subluxado, com implante primário de LIO, é um procedimento viável e que, quando bem indicado, pode melhorar bastante a acuidade visual destes pacientes. É importante salientar a necessidade de uma avaliação pré-operatória completa, com especial atenção à periferia da retina em pacientes com síndrome de Marfan. Além disso, só o conhecimento da técnica cirúrgica e dos recursos disponíveis no tratamento destas patologias permite oferecer a esses pacientes uma opção segura de reabilitação visual.

\section{ABSTRACT}

Purpose: To evaluate 10 cases of subluxated lens due to trauma or Marfan syndrome, focussing on their treatment and the results. Methods: Ten eyes of 9 patients were operated on due to lens subluxation. Six eyes were due to trauma and 4 eyes to Marfan syndrome. The mean age was $48.9 \pm 18.2$ years, and the follow-up period of $34.6 \pm 11.6$ months. Patients were submitted to intracapsular lens extraction (1 eye), extracapsular lens extraction (1 eye), phacoemulsification without IOL (1 eye), and phacoemulsification with IOL ( 7 eyes). Endocapsular rings were used in 7 eyes. Results: Best-corrected visual acuity improved in all patients, except for one, who had a regmatogeneous retinal detachment, 20 days after surgery. Conclusion: Lens extraction with IOL implantation in subluxated lens patients has a good prognosis, especially with phacoemulsification and endocapsular rings. With this approach, we were able to improve visual acuity and life quality in $90 \%$ of the cases.

Keywords: Lens subluxation/surgery; Eye injuries; Marfan syndrome; Cataract extraction

\section{REFERÊNCIAS}

1. Kanski JJ. Ectopia lentis. In: Kanski JJ. Clinical Ophthalmology: a systematic approach. $3^{\text {rd }}$ ed. London: Butterworths;1994. p.307-9

2. Blum M, Tetz MR, Greiner C, Voelcker HE. Treatment of traumatic cataracts. J Cataract Refract Surg 1996;22:342-6.

3. Streeten BW. Pathology of the Lens. In: Albert DM, Jakobiec FA. Principles and practice of ophthalmology: clinical practice. Philadelphia: WB Saunders; 1994. p.2180-238. 
4. Halpert M, BenEzra D. Surgery of the hereditary subluxated lens in children. Ophthalmology 1996;103:681-6.

5. Moreira H, Miranda E, Bazzi GF. Catarata traumática. In: Moreira Jr CA, Freitas D, Kikuta HS. Trauma ocular. Rio de Janeiro: Cultura Médica;1997. p.46-7.

6. Zetterström C, Lundvall A, Weeber H, Jeeves M. Sulcus fixation without capsular support in children. J Cataract Refract Surg 1999;25:776-81.

7. Lam DS, Ng JS, Fan DS, Chua JK, Leung AT, Tham CC. Short-term results of scleral intraocular lens fixation in children. J Cataract Refract Surg 1998; 24:1474-9.

8. Legler U, Witschel B, Lim S, et al. The capsular ring: a new device for complicated cataract surgery. Symposium on Cataract, IOL and Refractive Surgery: Seattle; 1993.
9. Cionni RJ, Osher RH. Management of profound zonular dialysis or weakness with a new endocapsular ring designed for escleral fixation. J Cataract Refract Surg 1998;24:1299-306.

10. Cionni RJ, Osher RH. Endocapsular ring approach to the subluxed cataractous lens. J Cataract Refract Surg 1995;21:245-9.

11. Adank AM, Hennekes R. Phacoemulsification of the subluxated or atopic lens. Bull Soc Belge Ophtalmol 1993;249:33-9.

12. Vadalà $\mathrm{P}$, Capozzi $\mathrm{P}$, Fortunato $\mathrm{M}$, DeVirgiliis E, Vadalà F. Intraocular lens implantation in Marfan's syndrome. J Pediatr Ophthalmol Strabismus 2000; 37:206-8.

13. Lam DS, Young AL, Leung AT, Rao SK, Fan DS, Ng JS. Scleral fixation of a capsular tension ring for severe ectopia lentis. J Cataract Refract Surg 2000;26:609-12.

\title{
IV CONGRESSO DA SOCIEDADE CAIPIRA DE OFTALMOLOGIA
}

\author{
2 e 3 de julho de 2004 \\ Centro de Convenções \\ do Hospital das Clínicas de \\ Ribeirãe Prete - SP
}

\section{Informações e Inscrições: CENACON \\ Tel.: (17) 235-7017/Fax: (17) 235-5334 \\ E-mail: cenacon@cenacon.com.br \\ Home-page: www.cenacon.com.br}

